\title{
Abstracts from the 2nd International Norges Teknisk- Naturvitenskapelige Universitet (NTNU) Symposium: Day 1-Immunotherapy and Hematology
}

\author{
Current and Future Clinical Biomarkers of Cancer: From diagnosis to immunotherapy-why is \\ precision medicine so difficult? \\ 14-15 June 2018, Trondheim, Norway
}

Published online: 7 October 2019

(c) Springer Nature Limited 2019

Experts from Europe, USA, China, Japan and Australia shared their recent work in presentations (https://vimeo.com/showca se/5456812).

Organizers: Oluf Dimitri Røe, Magne Børset, Anders Sundan, Anne-Marit Sponaas

Disclosures: Oluf Dimitri Røe is funded in part by the Liaison Committee for the Central Norway Regional Health Authority (RHA) and the NTNU and has received an honorarium for one lecture at a scientific meeting arranged by Novartis. The other organizers have no financial disclosures.

This Symposium aims at presenting research on clinically useful biomarkers and emerging concepts of diagnosis, prognosis, prediction and treatment of cancer. These abstracts mainly from Day 1 focused on hematological malignancies and many aspects of clinical immunotherapy biomarkers, immunotherapy modulation, microbiome and toxicity, as well as financial toxicity.

Sponsorship: The symposium was sponsored by the Norwegian University of Science and Technology (Norges TekniskNaturvitenskapelige Universitet, NTNU), HUNT (Helse-undersøkelsen i Nord-Trøndelag) research center, the Norwegian Research Council and Immunological Society of Norway, Norwegian Society of Biochemistry, Norwegian Cancer Society and AstraZeneca, BMS, MSD, Amgen, Celgene, Pfizer, Roche and EISAI. All content was reviewed and approved by the authors and organizers, which held full responsibility for the abstract selections. Publication of the supplement was sponsored by Norwegian University of Science and Technology (NTNU).

\section{E01}

Mitochondrial manipulation improves the PD-1 blockade immunotherapy

\section{Kenji Chamoto ${ }^{\text {, Tasuku Honjo }}{ }^{1}$ \\ ${ }^{1}$ Kyoto University, Kyoto, Japan}

From the Kyoto University, Japan, and the scientific group of recent Nobel Prize winner Tasaku Honjo (that discovered the PD-1), associate professor Kenji Chamoto presented studies on resistance to PD-1 inhibitors through immune metabolism. Professor Chamoto reported that tumor- reactive cytotoxic $\mathrm{T}$ lymphocytes (CTLs) in draining lymph nodes (DLNs) of PD-1 ${ }^{-1-}$ mice have activated mitochondria, and that mitochondrial perturbation chemicals had synergistic effects with a PD-1-blockade antibody in a mouse tumor model (1). In this model, mitochondrial activation was mediated by a key mitochondrial regulator, peroxisome proliferator-activated receptor gamma coactivator 1-alpha (PGC-1 $\alpha)$. They next targeted a peroxisome proliferator-activated receptor (PPAR), one of the transcription factors conjugated with PGC- $1 \alpha$ and found that a PPAR agonist, benzafibrate synergistically increased the effect of the PD-1 blockade therapy. Benzafibrate activated not only mitochondria, but also upregulated the overall 
metabolic state in CTLs, and promoted the proliferation of CTLs. In addition, benzafibrate augmented fatty acid oxidation and expression of carnitine palmitoyl transferase 1 (Cpt1), which stabilizes B-cell lymphoma 2 (Bcl2) expression, leading to the prevention of PD-1 blockade-induced apoptosis in killer $\mathrm{T}$ cells. In conclusion, benzafibrate increased the number of functional CTLs by increased proliferation and decreased apoptosis of CTLs via reprogramming of mitochondrial metabolism, and enhanced efficacy of PD-1 blockade. These findings should pave a way to develop novel combinatorial therapies with PD-1 blockade which bridge energy metabolism and $\mathrm{T}$ cells immunity (2).

Disclosure: There are no competing interests. $\mathrm{KC}$ and $\mathrm{TH}$ hold patents in PD1-blockade immunotherapy; $\mathrm{KC}$ and $\mathrm{TH}$ has received grant support from Bristol-Myers Squibb, Ono Pharmaceutical Co, Ltd, and Sysmex Corporation. TH has received grant support from Japan Agency for Medical Research and Development.

\section{References}

1. Chamoto K, Chowdhury PS, Kumar A, Sonomura, K, Matsuda F, Fagarasan, S, et al. Mitochondrial activation chemicals synergize with surface receptor PD-1 blockade for T cell-dependent antitumor activity. Proc Natl Acad Sci USA. 2014;114:E761-E770.

2. Chowdhury PS, Chamoto K, Kumar A, Honjo T. PPAR-induced fatty acid oxidation in T cells increases the number of tumor-reactive $\mathrm{CD} 8(+) \mathrm{T}$ cells and facilitates anti-PD-1 therapy. Cancer Immunol Res. 2018.

\section{E02}

Tissue residence and innate immunity: opportunities for new approaches to improve immunotherapy of cancer

\section{Madhav V. Dhodapkar ${ }^{1}$ \\ ${ }^{1}$ Emory University School of Medicine, Atlanta, GA, USA}

Antibody-mediated blockade of inhibitory immune checkpoints CTLA-4 and PD-1 has led to durable regression in several cancer types (1). However, these checkpoints/ molecules are expressed only on a small subset of immune cells infiltrating human tumors; understanding the biology of these cells may help optimize clinical application of these therapies. Professor Dhodapkar showed that the expression of PD-1 and some other immune checkpoints in melanoma lesions were enriched within the subset of $\mathrm{T}$ cells that carry features of tissue resident memory (TRM) T cells (2). The TRM cells can persist in tissues for decades without recirculation (3). Accordingly, they contribute to inter-lesional heterogeneity of $\mathrm{T}$ cell receptors within individual metastatic lesions and do not equilibrate between lesions in spite of highly overlapping neoantigenic load. Tissue residence is also a feature of several innate immune cells such as natural killer $\mathrm{T}$ (NKT) cells that recognize lipid antigens in the context of CD1d. CD1d is expressed by several hematologic malignancies, which are an attractive target of therapies utilizing NKT cells. His research showed that combination approaches targeting NKT cells lead to durable regression in early myeloma lesions (4). Harnessing properties of tissue-resident innate and adaptive immune cells has the potential to improve immune-mediated control in several cancers.

Disclosure: MV has received consulting fees or served as a paid advisory board member at Amgen, Kite, Roche, Lava Therapeutika, Celgene and Janssen.

\section{References}

1. Ribas A, Wolchok JD. Cancer immunotherapy using checkpoint blockade. Science. 2018;359:1350-5.

2. Boddupalli CS, Bar N, Kadaveru K, Krauthammer M, Pornputtapong N, Mai Z, et al. Interlesional diversity of $\mathrm{T}$ cell receptors in melanoma with immune checkpoints enriched in tissue-resident memory $\mathrm{T}$ cells. JCI Insight. 2016;1:e88955.

3. Sathaliyawala T, Kubota M, Yudanin N, Turner D, Camp P, Thome JJ, et al. Distribution and compartmentalization of human circulating and tissue-resident memory T cell subsets. Immunity. 2013;38:187-97.

4. Richter J, Neparidze N, Zhang L, Nair S, Monesmith T, Sundaram R, et al. Clinical regressions and broad immune activation following combination therapy targeting human NKT cells in myeloma. Blood. 2013;121: 423-30.

\section{E03}

Immune checkpoint blockade in multiple myeloma: can we reinvigorate without harm?

\section{Alexander Lesokhin ${ }^{1}$}

${ }^{I}$ Memorial Sloan Kettering Cancer Center, New York, NY, USA

Doctor Lesokhin presented various aspects and problems on PD-1/PD-L1 treatment in multiple myeloma. Whilst monotherapies with PD-1/PD-L1 gave little clinical activities (1), phase I and IIb trials combining standard treatment with low dose dexamethasone and immunomodulating drugs (iMiDs) such as lenalidamide/pomalidomide gave promising results. In two studies, one with lenalidomide in patients advanced, refractory cancers and another one with 
pomalidomide gave a $50 \%$ and $60 \%$ response rate respectively. These studies led to phase III studies in relapsed and refractory as well as previously untreated patients. Both trials were however terminated prematurely due to more deaths in the pembrolizumab arm. Some of these deaths appeared to be of an immunological nature. In addition, no clinical benefit was observed in the patients receiving pembrulizumab and iMiDs. This led to the FDA stopping all trials with anti-PD-1 pathway blocking agents that contained an IMiD drug or where the PD-1 blocking agent was being evaluated in non-relapsed, refractory patients. These results have led to a discussion among clinicians and scientists on how to move on from this disappointment. Lesokhin pointed out that it is now very important to study the immune biology in myeloma patients and consider other drugs, combinations or therapies such as chimeric antigen receptor T-cell therapy (CAR-T), engineered antibodies, and vaccines. He also highlighted the role that PD-L1 can play in myeloma disease, e.g. plasma cells from relapsed patients with advanced disease and plasma cells from MRD + patients with poor prognosis express very high levels of PD-L1. Lesokhin emphasized that it is very important to study immune cells in the bone marrow of myeloma patients and presented some very exiting unpublished data (submitted for publication) suggesting that in some patients, a subset of activated $\mathrm{T}$ cells in the bone marrow after autologous bone marrow transplant was associated with inferior outcomes.

Disclosure: AL has received consulting fees from BristolMyers Squibb and Genmab, lecture fees from Takeda, and grant support from Bristol-Myers Squibb and Janssen.

\section{Reference}

1. Lesokhin AM, Ansell SM, Armand P, Scott EC, Halwani A, Gutierrez M, et al. Nivolumab in patients with relapsed or refractory hematologic malignancy: preliminary results of a phase Ib study. J Clin Oncol. 2016;34:2698-2704.

\section{E04}

\section{V(D)J clonality detection for DNA-based tracking of minimal residual disease in multiple myeloma}

\section{Even H. Rustad ${ }^{1}$}

${ }^{1}$ Memorial Sloan Kettering Cancer Center, New York, NY, USA

Achieving minimal residual disease (MRD) negativity after first-line treatment is a strong predictor of favorable outcomes in multiple myeloma. Molecular MRD assays are increasingly being adopted, based on tracking of tumor-specific clonal immunoglobulin, variable, diversity and joining genes (V(D) $\mathrm{J})$ rearrangement sequences by next generation sequencing. However, previous studies have reported failure to identify the clonal V(D)J sequence in 5 to 20 percent of patients, which may limit applicability. Rustad and colleagues presented their study designed to define the sample-related and disease-related factors influencing V(D)J clonality detection. Baseline bone marrow samples from 177 patients with plasma cell myeloma were included. These samples from their research tissue bank were drawn after the initial bone marrow aspirate volume was taken aside for clinical use. Baseline characterization of $\mathrm{V}(\mathrm{D}) \mathrm{J}$ sequence clonality was done using the LymphoTrack ${ }^{\circledast}$ VDJ assays (Invivoscribe, Inc, San Diego, CA). As a molecular control for detectable tumor-derived DNA in the samples, they sequenced the same samples using their in-house myeloma panel myTYPE. Results from their research samples were compared with a cohort of clinical samples from their institution, where the same V(D)J assays were used as part of routine workup. They identified a clonal $\mathrm{V}(\mathrm{D}) \mathrm{J}$ sequence in $81 \%$ in their whole cohort of research samples, compared to $95 \%$ in samples where tumor DNA was detected by myTYPE (i.e. one or more putative somatic single nucleotide variant, insertion, deletion, translocation or copy number variation). In a multivariate logistic regression model, they identified three independent predictors of successful $\mathrm{V}$ (D)J clonality detection: (I) myTYPE positivity (odds ratio [OR] 5.56), (II) bone marrow plasma cell (BMPC) percentage on aspirate smear (OR 1.44 per $10 \%$ increase), (III) and lambda light chain restriction (OR 7.09).

In clinical samples, the success rate of $\mathrm{V}(\mathrm{D}) \mathrm{J}$ clonality detection was $96 \%$ when BMPC infiltration by aspirate smear was $>5 \%$. They concluded that failure to identify a clonal $\mathrm{V}$ (D)J sequence was most commonly due to poor sample quality with low plasma cell content. Comparison between research samples and clinical samples points to hemodilution as the main cause. Importantly, they showed that high rates of baseline VDJ capture around 95\% can be achieved if bone marrow sampling and processing is optimized for plasma cell yield.

Disclosure: ER has no competing interests.

\section{E05}

The role of ectoenzymes CD39 and CD73 in the immune response to multiple myeloma

\section{Rui Yang $^{1}$, Anne-Marit Sponaas ${ }^{1}$ \\ ${ }^{I}$ Norwegian University of Science and Technology (NTNU), Trondheim, Norway}

The authors presented novel evidence from their group showing bone marrow levels of adenosine might represent a 
new immune checkpoint in bone marrow of patients with multiple myeloma. It is well known that extracellular adenosine may suppress for example $\mathrm{T}$ cell activity, and by analyzing bone marrow aspirates from multiple myeloma patients, Yang found that the patients had significantly elevated levels of adenosine compared to healthy controls. She showed how adenosine could be generated from ATP through the activity of the ectoenzyme CD39 expressed on myeloma cells which converts ATP to AMP. The AMP could subsequently be converted to adenosine by another extoenzyme, CD73, which is expressed on bone marrow stromal cells. Furthermore, Yang showed that she could reconstitute this biochemical pathway in vitro by co-culture experiments using myeloma cells and stromal cells, as well as T cells. Finally, she demonstrated that the anti-proliferative effect of adenosine on $\mathrm{T}$ cells was mediated by the A2 adenosine receptor. Taken together, her work identified several putative targets for clinical intervention with the aim of counteracting the immunosuppressive activity of extracellular adenosine in myeloma bone marrow. Yang also became the recipient of the Best Abstract Award.

Disclosure: RY and AMS have no competing interests.

\section{E06}

\section{DNA sensing and tryptophan catabolism in the tumor microenvironment}

\author{
Andrew Mellor', Lei Huang ${ }^{1}$, Henrique Lemos ${ }^{1}$ \\ ${ }^{1}$ Newcastle University, Newcastle, UK
}

Immune checkpoints are hallmarks of the tumor microenvironment (TME) at clinical presentation and pathways that suppress $\mathrm{T}$ cell responses are targeted with monoclonal antibodies to disrupt these. However, another common TME checkpoint involves elevated tryptophan (Trp) catabolism mediated by cells expressing indoleamine 2,3 dioxygenase (IDO). Professor Andrew Mellor, Newcastle University, UK, showed that small molecule IDO inhibitors are under scrutiny as potential drugs to disrupt IDOmediated immune checkpoints and enhance anti-tumor immunity (1). Despite major focus on disrupting immune checkpoints, it is unclear how they arise during tumourigenesis. To address this question, they assessed IDO induction during growth of Lewis Lung Carcinoma in mice (2). Lewis Lung Carcinoma engraftment induced rapid increase in IDO activity in local lymph nodes draining and dendritic cells were the major cell type to express IDO. This response was dependent on DNA sensing to activate the Stimulator of Interferon Genes (STING) adapter. Optimal Lewis Lung Carcinoma tumor growth also depended on STING and IDO, indicating that dying cells in the aseptic TME released DNA to promote tumor growth via STING/ IFN-I signaling to induce IDO. In contrast, LLC tumor cells expressing neo-antigens grew faster in mice lacking STING genes, indicating that DNA sensing inhibited tumor growth in this model. Their findings revealed that DNA sensing is a pivotal pathway in the TME that promotes or suppresses immunity contingent on context and tumor immunogenicity. Thus, immune responses to DNA are key factors influencing immune balance that drives or impedes tumor growth as well as responses to therapy (Fig. 1). Elevated IDO activity in the TME may also drive neurologic

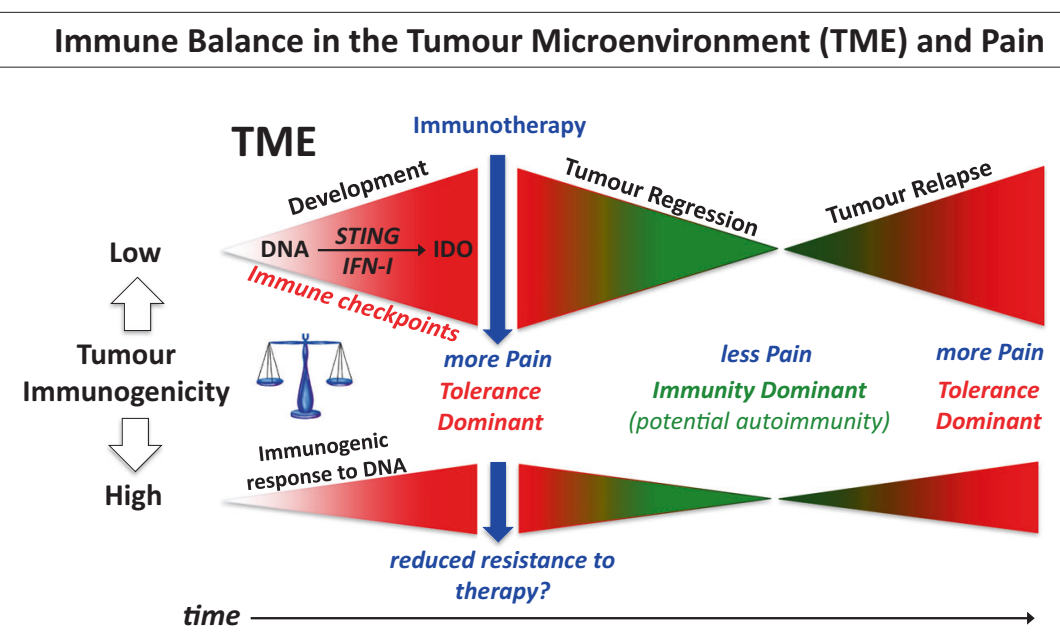

Figure 1 [E06] DNA impacts immune balance and tumor growth. Dying cells in the developing Tumor Microenvironment (TME) release DNA, which is sensed to induce IDO and suppress anti-tumor immunity (red highlights). Immunotherapy incites anti-tumor immunity (green highlights) to promote tumor regression but DNA driven immune suppression may re-establish after therapy. Increased pain is also a potential consequence of elevated IDO activity in the tolerogenic TME. Elevated tumor immunogenicity may reduce barriers to therapy by enhancing immunogenic responses to DNA in the TME. 
comorbidities associated with cancer or cancer therapy such as pain, depression and fatigue since cells expressing IDO release neuro-active catabolites (3). In ongoing studies, they also found that tumor growth and therapy with STING agonists heighten pain hyper-sensitivity. In principle, the use of IDO inhibitors offers novel ways to disrupt immune checkpoints and reduce neurologic comorbidities that affect cancer patients.

Disclosure: AM has stock options in NewLink Genetics Inc., and is an inventor on 19 patents held by Augusta University GA USA. AM has received licence payments on some of these patents until last year via Augusta University, and served as an expert witness in November 2018. AM and LH have received grant support from Cancer Research UK (CRUK). LH and HL have no competing interests.

\section{References}

1. Lemos H, Huang L, McGaha T, Mellor AL. STING, nanoparticles, autoimmune disease and cancer: a novel paradigm for immunotherapy? Expert Rev Clin Immunol. 2015;11:155-65.

2. Lemos H, Mohamed E, Huang L, Ou R, Pacholczyk $\mathrm{G}$, Arbab AS, et al. STING promotes the growth of tumors characterized by low antigenicity via IDO activation. Cancer Res. 2016;76:2076-81.

3. Huang L, Ou R, Rabelo de Souza G, Cunha TM, Lemos H, Mohamed E, et al. Virus infections incite pain hypersensitivity by inducing indoleamine 2,3 dioxygenase. PLoS Pathog. 2016;12:e1005615.

\section{E07 \\ Role of B cells in autoimmune complications following combination checkpoint blockade}

\section{Kavita Dhodapkar}

${ }^{l}$ Emory University School of Medicine, Atlanta, GA, USA

Combination checkpoint therapy with anti-CTLA-4 and anti-PD-1 leads to higher response rates against melanoma than monotherapy with either anti-CTLA-4 or anti-PD-1, but also to increased frequency of serious (grade 3 and 4) immune-related adverse events (IRAEs). Measures to predict and prevent the risk of IRAE are currently lacking. Kavita Dhodapkar, associate professor of pediatrics at Emory University, Atlanta, Georgia, presented their work, studying the changes in various immune cell populations in melanoma patients treated with these therapies, either as monotherapy or in combination. Unlike monotherapy, combination therapy significantly reduced the total level of circulating B cells, but increased and CD2 $1{ }^{\text {low }}$ subset of
B cells. These $\mathrm{CD} 21^{\text {low }} \mathrm{B}$ cells are enriched for memory cells, as they lack IgD and are hypermutated, suggesting that they are germinal-center-educated, antigen experienced cells (1). These cells are enriched in patients with autoimmune disorders and could lead to autoimmunity in patients treated with anti-CTLA-4/anti-PD-1 combination therapy. Dhodapkar showed that an early increase in the amount of circulating CD21 $1^{\text {low }} \mathrm{B}$ cells after combination checkpoint therapy predicted an increased risk of developing IRAE and this could potentially be used as a biomarker in disease monitoring. In addition, targeting these cells as a preemptive strategy before or during combination checkpoint therapy might be beneficial. Interestingly, whilst presence of $\mathrm{CD} 21^{\text {low }} \mathrm{B}$ cells correlated with severity of IRAE, changes in other subsets of circulating immune cells, T cells, myeloid cells and NK cells, did not correlate with the risk of autoimmunity $(1,2)$ uncoupling these two responses and opens up for the use of therapies targeting B cells to prevent IRAE in combination therapies.

Disclosure: KD has no competing interests.

\section{References}

1. Das R, Bar N, Ferreira M, Newman AM, Zhang L, Bailur JK,et al. Early B cell changes predict autoimmunity following combination immune checkpoint blockade. J Clin Investig. 2018;128:715-20.

2. Das R, Verma R, Sznol M, Boddupalli CS, Gettinger $\mathrm{SN}$, Kluger H, et al. . Combination therapy with antiCTLA-4 and anti-PD-1 leads to distinct immunologic changes in vivo. J Immunol. 2015;194:950-9.

\section{E08}

Targeting the gut microbiome in cancer immunosurveillance

\section{Conrad Rauber ${ }^{1}$, Laurence Zitvogel ${ }^{1,2,3}$}

${ }^{1}$ Gustave Roussy Cancer Campus (GRCC), Villejuif, France; ${ }^{2}$ Institut National de la Santé et de la Recherche Medicale (INSERM) and Equipe Labellisée-Ligue Nationale contre le Cancer, Villejuif, France ; ${ }^{3}$ Université ParisSud, Université Paris-Saclay, Gustave Roussy, Villejuif, France

Anticancer therapies influence the tumor microenvironment, but the response to anticancer treatment such as checkpoint therapy is also dependent on many other factors, one of which is the gut homeostasis. Doctor Rauber showed that a deviated repertoire of the intestinal microbiome called "dysbiosis", caused by the use of broad spectrum antibiotics, compromised the efficacy of conventional 
chemotherapy, as well as, immune checkpoint treatment in cancer patients.

The experiment presented used fecal samples from patients collected before PD-1 inhibitor treatment. These were transplanted into the intestines of $\mathrm{C} 57 \mathrm{Bl} / 6$ mice previously injected with the syngeneic MCA205 tumor. Interestingly, transplanting fecal microbiota from cancer patients responding to immunotherapy into germ-free or antibiotic-treated mice allow the antitumor effects of PD-1 blockade. Conversely, transplantating fecal microbiota from non-responding patients blocked the effects of anti-PD1 Abs (1). Metagenomics of patient stool samples at diagnosis showed correlation of therapeutic response with the presence of the commensal Akkermansia muciniphila.(1) Importantly, oral delivery of Akkermansia muciniphila partly restored the efficacy of PD-1 blockade via the recruitment of CCR9+CXCR3 $+\mathrm{CD} 4+\mathrm{T}$ lymphocytes. In conclusion, they found that antibiotics inhibited the clinical benefit of PD-1 blockade in patients with advanced cancer and that precise modification of the gut microbiome holds great promise in ameliorating patient's response to ICB therapy (1).

Disclosure: LZ is founder of everImmune and serves as a board member of Lytix Biopharma. LZ received lecture fees from AstraZeneca and Kiwamu Otsubo, Secretariat General-4th NCCH Workshop, National Cancer Center, receive research funding from GlaxoSmithKline, Lytix Biopharma, Merus, Roche, EpiVax, Incyte, Bristol-Myers Squibb, Innovate Pharma, Pileje and Transgene. LZ also hold a patent for everImmune. CR has no competing interests.

\section{Reference}

1. Routy B, Le Chatelier E, Derosa L, Duong CPM, Alou MT, Daillere R, et al. Gut microbiome influences efficacy of PD-1-based immunotherapy against epithelial tumors. Science. 2018;359:91-7.

\section{E09 \\ Opportunities for $\mathbf{T}$ cell receptor-based cancer immunotherapy}

\section{Johanna Olweus ${ }^{1}$}

${ }^{1}$ University of Oslo and Head of Department of Cancer Immunology at Oslo University Hospital Radiumhospitalet, Oslo, Norway

Although checkpoint inhibition has revolutionized the treatment of metastatic solid cancer, the majority of patients eventually relapse and die due to insufficient $\mathrm{T}$ cell responses. Possible explanations include immune tolerance, or ignorance due to ineffective presentation of neo-antigens to $\mathrm{T}$ cells. This talk focused on strategies to use the $\mathrm{T}$ cell repertoires of healthy donors to support the immune response of the patient. While tumors harbor a large number of mutated proteins, it is now clear that only $1-3 \%$ of these are recognized by intra-tumoral $\mathrm{T}$ cells. Olweus'group has recently shown that this can be overcome by immune "outsourcing". Thus, neo-antigens neglected by the patient's immune system can be "seen" by $\mathrm{T}$ cells from healthy individuals used to obtain reactive TCRs. Genes encoding the TCRs can be transferred to patient $\mathrm{T}$ cells and render them capable of killing tumor cells. The majority of neoantigens are, however, unique to the individual tumor. A limitation for potential therapeutic application of $\mathrm{T}$-cell receptor-mediated targeting is thus the need for identification of reactive $\mathrm{T}$-cell receptors for each patient. To overcome the need to target neo-antigens, the group has designed an alternative strategy. By establishing methods to identify $\mathrm{T}$ cells that recognize peptides from wild type proteins with cell typerestricted expression ${ }^{2}$, a single TCR can be used to treat a large number of patients. It is worth noting that all therapeutic antibodies (e.g. anti-CD20 for lymphoma) target "normal" cell surface proteins with cell type-restricted expression. The number of candidate TCR targets is manifold higher since TCRs can "see" peptides derived from anywhere in the cells. T-cells that can bind with high affinity to self-antigens presented on self-MHC are, however, removed by central tolerance in the thymus. The group of Olweus has demonstrated that $\mathrm{T}$ cells from healthy donors can provide a rich source of $\mathrm{T}$-cell receptors that efficiently kill specific cell types by recognition of cell-type restricted self-peptides in complex with foreign $\mathrm{HLA}^{3}$. Taken together, the results from the group indicate that donor-derived T-cell receptor repertoires could be utilized to overcome some of the limitations of host $\mathrm{T}$ cells in future cancer immunotherapy.

Disclosure: JO is on the Scientific Advisory Board of Intellia, receive funding and has a research collaboration with Kite Pharma.

\section{References}

1. Stronen E, Toebes M, Kelderman S, van Buuren MM, Yang W, van Rooij N, et al. Targeting of cancer neoantigens with donor-derived $\mathrm{T}$ cell receptor repertoires. Science. 2016.

2. Kumari S, Walchli S, Fallang LE, Yang W, LundJohansen F, Schumacher TN, et al. Alloreactive cytotoxic $\mathrm{T}$ cells provide means to decipher the immunopeptidome and reveal a plethora of tumorassociated self-epitopes. Proc Natl Acad Sci USA. 2014;111:403-8. 
3. Mensali N, Ying F, Sheng VO, Yang W, Walseng E, Kumari S, et al. Targeting B-cell neoplasia with $\mathrm{T}$ cell receptors recognizing a CD20-derived peptide on patient-specific HLA. Oncoimmunology. 2016;5: e1138199.

\section{E10}

\section{Cancer immunotherapy using a novel nanoparticle mRNA delivery platform}

\author{
Ole Audun Werner Haabeth ${ }^{1}$, Timothy Blake ${ }^{I}$ \\ ${ }^{1}$ Stanford University, Stanford, CA, USA
}

Immunotherapy by injecting antigen-encoding mRNA is a promising approach to personalized cancer treatment. Doctor Ole Audun Werner Haabeth presented their work representing Professor Ronald Levy's group (Stanford University, CA, USA) where they have developed of a novel, versatile, and highly efficient mRNA delivery system that employs Charge-Altering Releasable Transporters (CARTs) $(1,2)$. With this platform, they are able to deliver mRNAs encoding antigen or functional genes to antigen-presenting cells (APCs) and $\mathrm{T}$ cells upon intravenous and sub cutaneous injections. Upon local injections into the tumor, they observed an efficient gene delivery to tumor cells as well as tumor infiltrating cells. In a multi-group collaboration at Stanford University they are exploring the use, and optimization of this platform for various therapeutic approaches to treat cancer. They concluded that this platform can be used for cancer vaccination demonstrating that local gene-delivery can induce durable systemic responses (3) as well as gene therapy targeted to the effector function of endogenous T cells.

Disclosure: Both authors have filed a Charge-Altering Releasable Transporters (CART) patent.

\section{References}

1. McKinlay CJ, Vargas JR, Blake TR, Hardy JW, Kanada M, Contag $\mathrm{CH}$, et al. (Charge-altering releasable transporters (CARTs) for the delivery and release of mRNA in living animals. Proc Natl Acad Sci USA. 2017;114:E448-456.

2. McKinlay CJ, Benner NL, Haabeth OA, Waymouth RM, Wender PA. Enhanced mRNA delivery into lymphocytes enabled by lipid-varied libraries of charge-altering releasable transporters. Proc Natl Acad Sci USA. 2018;115:E5859-66.

3. Haabeth OAW, Blake TR, McKinlay CJ, Waymouth RM, Wender PA, Levy R. mRNA vaccination with charge-altering releasable transporters elicits human
$\mathrm{T}$ cell responses and cures established tumors in mice. Proc Natl Acad Sci USA. 2018;115: E9153-61.

\section{E11}

The cost of new cancer drugs, ethics and health disparities

\section{Oluf Dimitri Røe $e^{1,2,3}$}

${ }^{1}$ Levanger Hospital, Nord-Trøndelag Health Trust, Levanger, Norway; ${ }^{2}$ Norwegian University of Science and Technology, Trondheim, Norway; ${ }^{3}$ Aalborg University Hospital, Aalborg, Denmark

Access to life-saving or life-prolonging medicine is not equally distributed. There are disparities both on access to treatment between rich and poorer countries but also an uneven distribution within countries. These inequalities have their root in several levels, the political system of a county (e.g. Scandinavian countries providing equal healthcare for all citizens versus USA with a privatized system), the economy of a country (e.g. health spending is less in rich than poor countries), and the personal economy of a patient (a rich patient can buy state-of the art treatment if the insurance do not provide it) as well as the pricing of the drugs. When the HIV epidemic came, and effective drugs where available, they were only afforded in high-income countries, whereas in countries with a high incidence, these medicines were unreachable (1). Similarly, cancer treatment is expensive, not only the drugs, the surgery, radiation therapy, repeat hospitalizations, infections, all add to the cost. Assistant Professor Røe discussed the issue of the uneven distribution and access to new, expensive cancer treatment. The author claimed that one of the key questions are purely ethical; Is health a human right or is it your own responsibility? In poor developing countries the answer is easy. If you are rich you may receive life-saving treatment. In a rich country not practicing the Hippocratic oath for all its citizens, such as the USA, the uninsured or poor will again be the losers. With health costs spiraling, even rich countries like Norway, which have coverage for all citizens including all hospital treatments and homebased treatments, now are facing a system of double standards, where again, the rich get access to the best treatments.

Disclosure: ODR has received an honorarium for one lecture at a scientific meeting arranged by Novartis and partly funded by the Liaison Committee for the Central Norway Regional Health Authority (RHA) and the NTNU. 


\section{Reference}

1. Roe OD. The high cost of new cancer therapies-a challenge of inequality for all countries. JAMA Oncol. 2017; 3:1169-70.

\section{E12}

\section{The long road to developing targeted therapy for cancer}

\section{Robert Peter Gale}

${ }^{1}$ Imperial College London, London, UK

Chronic myeloid leukemia (CML) is now curable using targeted therapy. How did this happen? In contrast to usual accounts of a breakthrough, in the media and from many scientists, the author traced the 200-year path to curing CML from the 1st clinical descriptions in the 19th century to current therapy. This path had had many unexpected twists and turns including studies of chicken sarcomas, oncogenes in mice, a notorious bank robber and chromosome abnormalities in humans (Fig. 1). The saga started with clinical descriptions, advances to our understanding of the biology, genetics, molecular biology and then bio- chemistry of CML and ended with the unexpected observation that these drugs can sometimes be stopped without CML recurring despite the certain persistence of CML stem cells (1). Many steps along the path could be anticipated, others were not so obvious and others a surprise (2). As seen repeatedly, and maybe it is a human trait, progress is attributed to one or a few persons, which also happened in the remarkable progress in curing CML. Dr Gale discussed the experimental basis of or this irrational bias in the work of psychologists Tversky and Kahneman (3, 4).

Disclosure: RPG is a part-time employee of the Celgene Corporation.

\section{References}

1. Fainstein E, Marcelle C, Rosner A, Canaani E, Gale RP, Dreazen O, et al. A new fused transcript in Philadelphia chromosome positive acute lymphocytic leukaemia. Nature. 1987;330:386-8.

2. Gale RP. Professor John M Goldman, 1938-2013: gentleman and scholar. Leukemia. 2014;28:1175-6.

3. Kahneman D. (Thinking, fast and slow. New York, NY, US: Farrar, Straus and Giroux; 2011.

4. Lewis, M. The undoing project: a friendship that changed our minds. New York: Nortin; 2017.
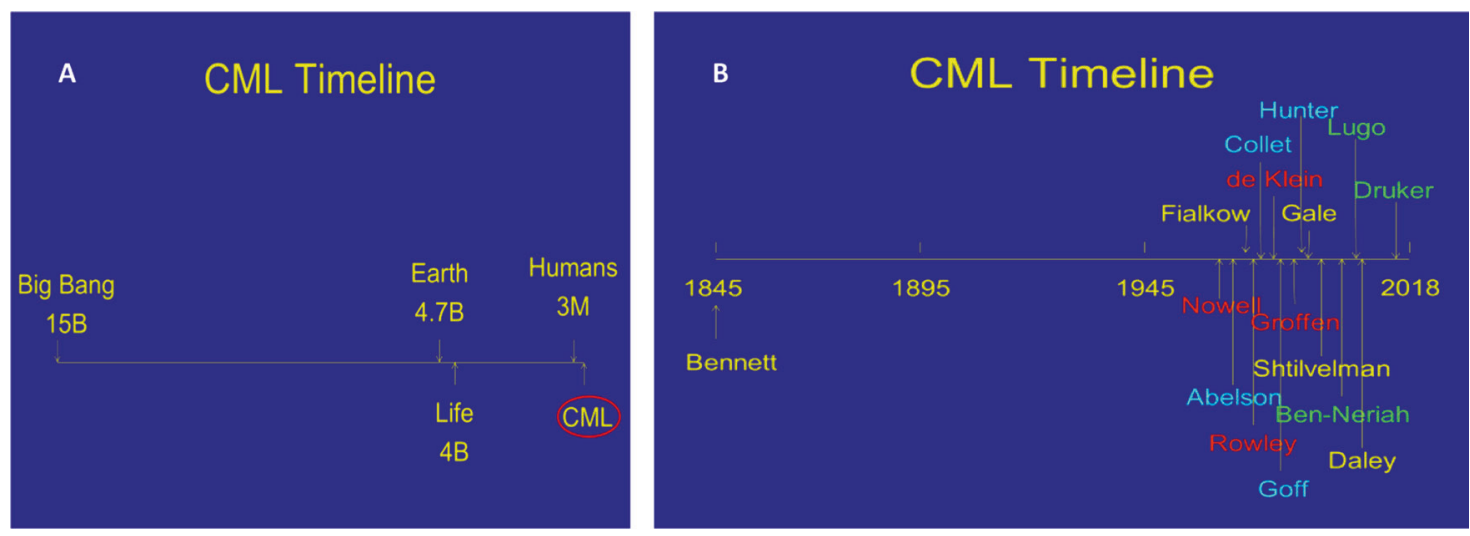

Figure 1 [E12] Time-line of chronic myeloid leukemia and targeted treatment in the perspective of the history of earth (a) and the last two centuries (b). 